23. Strümpell, Lehrbuch der speziellen Pathologie und Therapie, 1899. 24. Troisier, Société méd. des hôpiteaux. Sitzung vom 13. Januar 1899. Zentralbl. f. a. Pathologie, XI, pag. 581.

25. Wolf, K., Ober primären Lungenkrebs. Fortschr. der Medizin, 1895, Nr. 18 und 19 (Ref.).

26. Perrone, Cancroid in einer tub. Lungenkav., in Orth, Festschrift, Berlin, 1906.

\title{
XIII.
}

\section{Ein Fall von Lipom des Lig. latum.}

\author{
Von \\ Privatdozent Dr. Borrmann, \\ Prosektor am Herz. Krankenhaus in Braunschweig. \\ (Hierzu 1 Textfigur.)
}

Unter den s o li d e n Geschwülsten des breiten Mutterbandes nehmen die Fibrome und Fibromyome die erste Stelle ein, während andere, wie z. B. auch das Lipom, recht selten sind. So habe ich überhaupt nur einen Fall von Lipom des Lig. latum in der Literatur auffinden können, nämlich den von $\mathrm{Mi} d \mathrm{del} \mathrm{schulte}$ in einer Dissertation beschriebenen (Greifswald, 1884), auf den ich noch einmal kurz eingehen werde. Bei der Seltenheit dieser Fälle hielt ich es für angebracht, ein von mir zufällig bei der Sektion einer Geisteskranken in der Herzogl. Heil- und Pflegeanstalt in Königslutter gefundenes Lipom des breiten Mutterbandes zu veröffentlichen. Herr Dr. Fri e s e hat den Fall in einer soeben erschienenen Dissertation (Berlin, 1907) unter meiner Leitung verarbeitet, konnte jedoch die sehr instruktive Abbildung aus äußeren Gründen nicht mitdrucken lassen. Ich halte den Fall für wichtig genug, ihn nebst der Abbildung in der engeren Fachliteratur bekannt zu geben, und möchte noch einige Worte hinzufügen.

Der Tumor wurde, wie schon gesagt, bei der Sektion einer 59 jähr. Geisteskranken zufällig gefunden. Klinische Symptome hatte er nicht gemacht; einmal war auf die Angaben der Patientin nicht viel Wert zu legen und dann war der Tumor auch verhältnismäßig klein und brauchte keine Störungen zu verursachen. Er liegt auf der rechten Seite zwischen den Blättern des Lig. latum, (siehe Textfigur), ist $7 \frac{1}{2} \mathrm{~cm}$ lang, $6 \frac{1}{2} \mathrm{~cm}$ breit und $4 \mathrm{~cm}$ dick und besteht aus einem kleinen medianwärts und einem gröBeren lateralwärts gelegenen Knollen, die beide mehrfach gelappt sind. 
Interessant sind die Beziehungen des Tumors zu dem Lig. rotundum dextrum. Während letzteres auf der linken Seite normal entwickelt ist und normalen Verlauf zeigt, ist es auf der rechten Seite nur in seinem oberen Abschnitt entwickelt und hört dann auf, indem es auf der Höhe des Lipoms sich auffasert und im Lig. latum allmählich sich verliert.

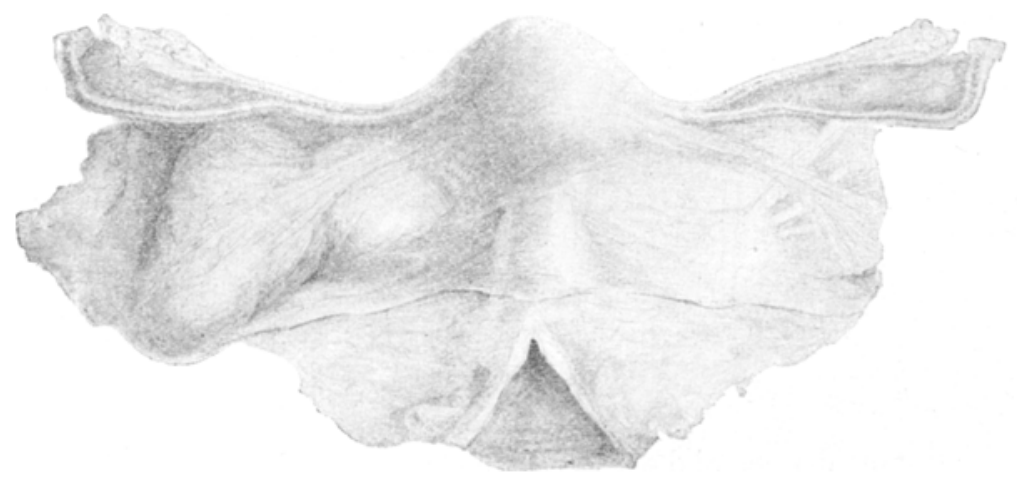

Entwicklungsgeschichtlich ist das Lig. rotundum eine Verdickung innerhalb einer von der Urniere nach unten gehenden Bauchfellfalte, aus der sich später das Lig. latum entwickelt. Das Lig. rotundum liegt also in $n$ e $\mathrm{r}$ h a l b des Lig. latum und verläuft dann weiterhin so, daß es an der Stelle, wo am Peritoneum eine kleine Ausstülpung, der processus vaginalis, sich findet, durch die Bauchwand hindurchtritt, um beim Weibe in der großen Schamlippe zu enden. In unserem vorliegenden Falle (vgl. Abbildung) zeigt das rechtsseitige Lig. rotundum nun eine Art Mißbildung, eine Anomalie, indem es schon nach einem Verlauf von etwa $6 \mathrm{~cm}$ unter allmählicher Auffaserung endet. Wir müssen also annehmen, daß das Band nicht vollkommen angelegt wurde, so daß nur sein oberer Abschnitt sich entwickelte; wir hätten also eine Art Hemmungsbildung. Nun ist weiterhin sehr interessant, daß gerade an jener Stelle, wo das Lig. rotundum im Lig. latum aufhört, das gelappte Lipom sich findet, und es ist naheliegend anzunehmen, daß die Störung in der Entwicklung des Lig. rotunđum zu einer weiteren Störung geführt hat, nämlich zur Abschnürung eines Fettgewebskomplexes, aus dem später dann das Lipom sich entwickelte. Man könnte auch vielleicht umgekehrt sagen: durch irgend eine Störung in der Entwicklung des Lig. latum kam es 
zur Abschnürung eines Fettgewebskomplexes, der dann wiederum dazu führte, daß hier die Anlage des Lig. rotundum ihr Ende erreichte. Im einzelnen wird sich ja natürlich nicht entscheiden lassen, welche Störung die erste war; jedenfalls kann man aber annehmen, daß sowohl die Lipomanlage als auch das Aufhören des Lig. rotundum auf ein und dieselbe Entwicklungsstörung zurückzuführen ist.

Um nun noch mit einigen Worten auf den Fall von $\mathrm{M}$ i d d e lschult e einzugehen, das einzige bisher veröffentlichte Lipom des Lig. latum, so sei erwähnt, daß der Tumor erhebliche klinische Erscheinungen gemacht hatte und sehr groß war: Gewicht $15 \mathrm{~kg}$, Größe 88:90 cm. Auch in diesem Falle wird erwähnt, daß das Lig. rotundum nach etwa $18 \mathrm{~cm}$ langem Verlauf quer über den Tumor allmählich aufhörte. (Das Ligament war natürlich in der Anlage nur ganz kurz und seine jetzige Länge von $18 \mathrm{~cm}$ dadurch zu erklären, daß es durch den wachsenden. Tumor ausgezogen wurde.) Man kann also auch für diesen Fall annehmen, daß eine Störung in der Anlage des Lig. rotundum vorhanden war und eventuell die Entstehung des Lipoms damit in Verbindung bringen, ähnlich wie in unserem Falle. Middelschulte geht auf diesen Punkt nicht näher ein. Die Fälle von Lipom des Lig. latum sind also einmal in differentialdiagnostischer Hinsicht nicht ohne Bedeutung und ferner scheinen sie auf entwicklungsgeschichtlichen Störungen $\mathrm{zu}$ beruhen, die mit einer unvollkommenen Anlage des Lig. rotundum im Zusammenhang stehen.

\section{XIV.}

Über den Bau, die eintretenden Gefäße und das Wachstum der warzenförmigen Angiome der Haut.

(Aus dem Pathologischen Institut der Universität Bonn.)

Von

Dr. S. $O$ g a w a

ans Japan.

Auf der äußeren Haut, zumal alter Individuen finden sich einzeln, besonders gern aber multipel die bekannten blau durch- 